\begin{tabular}{llllll} 
Abstract PTH-36 Table 1 & \\
\hline Group & $\begin{array}{l}\text { Total GI Clinic Rate/Year } \\
\text { Mean }(95 \% \text { Cl) }\end{array}$ & Colonoscopy Rate/Year & MRCP Rate/Year & USS Rate/Year & MRCP/USS Rate/Year \\
& $2.34(1.86-2.82)$ & $0.08(0.03-0.13)$ & $0.40(0.28-0.52)$ & $0.55(0.38-0.72)$ & $0.96(0.75-1.17)$ \\
PSC Only $(n=35)$ & $2.41(2.12-2.70)$ & $0.61(0.37-0.85)$ & $0.37(0.28-0.46)$ & $0.48(0.39-0.57)$ & $0.85(0.71-0.99)$ \\
PSC + IBD $(n=54)$ & 0.78 & $<0.001$ & 0.70 & 0.42 & 0.39 \\
P value & & &
\end{tabular}

radiology reports, endoscopy records and histology were extracted and digitally trawled to identify the cohort characteristics.

Results We identified 125 patients with PSC followed-up at UHS. $39.2 \%$ (49) of these patients were missed in a parallel criterion-based review of case notes.

We calculated an age-standardised point prevalence of 12.52 cases per 100,000 patients, $124 \%$ higher than typically cited UK figures. Service evaluation revealed high rates of clinic follow-up however lower than recommended rates of screening with colonoscopy and imaging (see Table 1). Introduction of a combined PSC/IBD clinic as a targeted service delivery intervention is addressing this shortfall with significant impact after 1 year.

Conclusions PSC cohorts are difficult to identify due to a lack of a UK clinical code. An NLP based methodology proved highly effective at identifying all cases within our institution, with a $64.5 \%$ increase compared to conventional methods. This allowed rapid patient cohort identification and conversion of unstructured data to clinically useful structured data and could be reproduced at other institutions and for other diseases.

\section{PTH-37 OVER INVESTIGATION IN PATIENTS WITH CHOLEDOCHOLITHIASIS: ROOM FOR SERVICE IMPROVEMENT?}

Alice Lagnado*, Charles Broome, Amy Woods, Shwan Karim, Chris Wadsworth. Hammersmith Hospital, London, UK

\subsection{6/gutjnl-2021-BSG.352}

Introduction Choledocholithiasis is the commonest reason for ERCP referrals, usually diagnosed with clinical history, blood results and imaging including ultrasound and cross sectional imaging if needed. The ESGE guidelines recommends three pathways in securing the diagnosis based on likelihood; low, intermediate and high. In those with intermediate risk, further imaging with MRI or EUS is recommended.

We noted that the majority of our referrals had multiple investigations including ultrasound, CT and MRCP prior to finally having a referral for ERCP. The aim of this study was to review our performance, evaluate feasibility of offering EUS in same session of ERCP instead of MRCP and formulate a service improvement plan aiming at reduced patients' length of stay and cost.

Methods All patients who had undergone an MRCP or ERCP while being an inpatient for possible biliary stone disease in a single tertiary referral centre between January 2018 and March 2020 were included. Data was retrospectively collected using our electronic patient record system and the ERCP database.

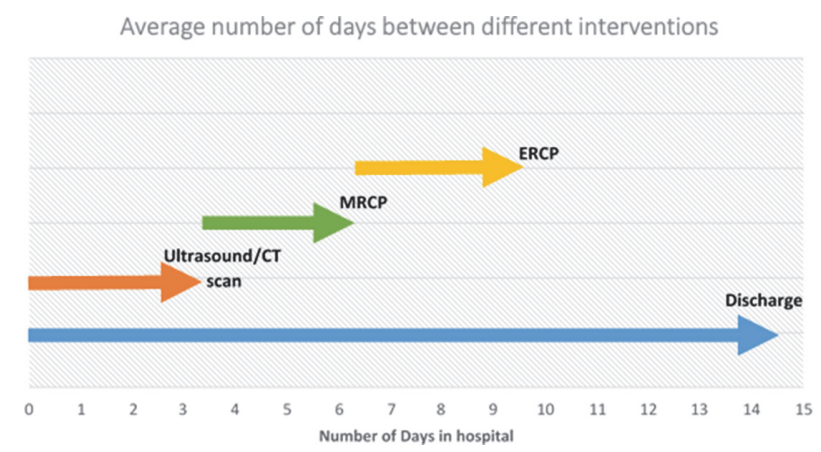

Abstract PTH-37 Figure 1 Timeline of patients' journey from admission to discharge

Results In total, 455 patients underwent an MRCP for possible stone disease. Of these, 157 had radiological evidence of choledocholithiasis and 129 went on to have an inpatient ERCP. The mean length of stay was 14.5 days (median 7, (1297)) and the average time from admission to ultrasound scan was 3.4 days (median 1 (0-92)) (Figure 1). There was an average of 2.9 days between USS/CT to MRCP (median 2 (0-23)), equivalent of a total of 1319.5 days over 26 months. For the patients who had an inpatient ERCP, there was an average of 3.4 days (median 2, (1-23)) between MRCP and ERCP, equivalent of 439 days over the 26 month period.

Conclusion and Plans Only 157 (34.5\%) of the MRCPs that were done for possible choledocholithiasis were positive in our cohort. The waiting times between procedures is correlated with an increase in LoS. In theory, if we had been able to offer these patients EUS (immediately pre ERCP) within $48 \mathrm{hrs}$ of admission, we would have saved approximately 848 inpatient hospital days over the 26months. However, our results demonstrate this would have created a large number of negative examinations, which is neither feasible nor cost effective.

As part of a service improvement project, we propose applying the ESGE updated guidelines using US findings and blood tests on admission, to scrutinise patients' pathways better, reduce number of ordered investigations (MRCPs in particular) and result in reduced length of stay, better use of resources and be more cost effective. Further research will be carried out to prove this in our cohort and will be reported separately.

\section{PTH-38 INDWELLING PERITONEAL CATHETER SERVICE FOR MALIGNANT RELATED ASCITES}

Avinash Aujayeb*. Northumbria Healthcare Nhs Foundation Trust, Newcastle, UK 
Introduction Traditional management of malignancy related ascites (MRA) is via large volume paracentesis (LVP). NICE recommends indwelling peritoneal catheters, (IPCs) which are cost effective and patient centred. A service and practice guidelines were set up. Ongoing reviews assess safety and efficacy and inform future pathways.

Methods A single centre retrospective analysis of patients requiring IPCs between October 2018 and December 2020 was performed. Demographics and outcomes were collected. Descriptive statistical methodology was applied. IPCs are placed in theatre or dedicated clean spaces, with pre-operative antibiotics.

Results 29 patients (14 male, 15 female) underwent 30 IPC placements. [1 patient had 2 IPCs (this will not be discussed as case is published)]. Diagnoses were, breast (2), ovarian (5), prostate (1), thymic (1), unknown primary (5) cancers and 1 mesothelioma. The majority were for gastrointestinal cancers. 3 had no preceding LVP; 26 had a mean of 2.2 LVPs before. 6 developed post-operative leaks. 2 patients developed cellulitis. Bacterial colonisation occurred in 1 patient. 4 IPCs were removed: 2 as ascites resolved, 1 for tumour infiltration, 1 for non-resolving site cellulitis. 24 patients have died: mean number of days to death: 60.1 (range 6-262). Integrated Palliative Care Outcome Scale scores collected in patients consistently show sustained reduction in pain, dyspnoea, nausea, vomiting, drowsiness, distension and improvements in body image and appetite.

Conclusions

IPCs are safe and efficient More formal evidence is required on some aspects namely a systematic review of the literature, a study to determine if patients with MRA should have an IPC at first presentation and correlate with quality of life outcomes and a national collaboration on numbers of IPCs being inserted and related outcomes.

\section{PTH-39 DELAYED DIAGNOSIS IN HEREDITARY HAEMOCHROMATOSIS - POTENTIAL UTILITY OF 'CASE- FINDING' APPROACH}

${ }^{1}$ Angus Kitchin* ${ }^{2}$ Sophie Stretch, ${ }^{1}$ Amin Abdulgader, ${ }^{1}$ Katharine Hutchison, ${ }^{1}$ Timothy Jobson. 'Somerset NHS Foundation Trust, Taunton, UK; ${ }^{2}$ Bristol University Medical School, Bristol, UK

\subsection{6/gutjnl-2021-BSG.354}

Introduction Hereditary haemochromatosis is a common genetic disorder, with a prevalence of $0.25-1 \%$ in the UK population. It has variable clinical penetrance, with men more frequently affected. Progression to liver cirrhosis can be prevented with early diagnosis and venesection. Increased testing of serum ferritin may lead to increased diagnosis, although haemochromatosis may still be overlooked, as elevated ferritin levels may be ascribed to malignancy or inflammatory disorders. If late diagnosis is common then a case-finding approach may identify patients earlier in their disease, with lower costs than a screening programme. The aim of this project was to identify whether patients with hereditary haemochromatosis are diagnosed within 6 months of an initial abnormal ferritin, and to estimate the number of patients that remain undiagnosed in Somerset.

Methods Somerset patients with haemochromatosis diagnosed between 2005-2020 were identified from clinic letters and venesection databases. Review of electronic blood results and clinic letters identified demographic data, time to diagnosis and ferritin result at diagnosis. For those with a delayed diagnosis (defined as over 6 months after a ferritin >500), the number of pre-diagnosis ferritin results was recorded.

Results 124 patients (30 female, 94 male) were identified. 83 patients $(20 \mathrm{~F}, 63 \mathrm{M})$ were diagnosed in Somerset after their first available ferritin estimation. 47 (56.6\%) were diagnosed within six months of their first raised ferritin. Of those with a delayed diagnosis, 9 (25\%) took over 3 years to be diagnosed and 4 (9\%) remained undiagnosed after 5 years. The higher the initial ferritin result, the more likely a patient was diagnosed within 6 months $(p=0.005)$. Using published data for the prevalence and clinical penetrance of genetic haemochromatosis, we predicted the expected number of patients with overt haemochromatosis in Somerset. From our modelling we would expect to see 72 women and 412 men with overt clinical haemochromatosis in our population. This indicates that in Somerset $58 \%$ of women and $77 \%$ of men with clinically relevant haemochromatosis may be undiagnosed.

Conclusions Our data show that diagnosis was delayed in almost half of Somerset patients with haemochromatosis. Additionally, it is a significantly underdiagnosed condition. Given that early initiation of venesection can reduce the risk of developing complications, improving the diagnostic pathway for patients with haemochromatosis using a 'case-finding' approach should be prioritised.

\section{PTH-40 PREVALENCE OF UNDIAGNOSED PRIMARY BILIARY CHOLANGITIS AND THE POTENTIAL UTILITY OF A 'CASE-FINDING' APPROACH}

${ }^{1}$ Katharine Hutchison*, ${ }^{1}$ Angus Kitchin, ${ }^{1}$ Amin Abdulgader, ${ }^{2}$ Sophie Stretch, ${ }^{1}$ Timothy Jobson. ' Somerset NHS Foundation Trust, Taunton, UK; ${ }^{2}$ Bristol University Medical School, Bristol, United Kingdon

\subsection{6/gutjnl-2021-BSG.355}

Introduction Primary Biliary Cholangitis (PBC) is a progressive, autoimmune disease for which treatment improves 10-year transplant free survival from $60 \%$ to $80 \%$. The observed variance in prevalence of PBC (1.91-40.2/100,000) may be in part explained by underdiagnosis. Liver function tests (LFTs) are frequently measured, but abnormalities are under-investigated. The purpose of this audit was to determine the rate at which abnormal alkaline phosphatase (ALP) results were investigated and whether patients meeting diagnostic criteria for $\mathrm{PBC}$ were referred in line with current guidelines. If existing laboratory data can identify missed $\mathrm{PBC}$, there is potential to use a case-finding approach.

Methods We interrogated our laboratory system (population of 500,000; 2011-2018) using the audit tool PathManager ${ }^{\text {TM }}$ to identify potential patients aged 40-60. Individuals with $>3$ abnormal ALP results, and $\geq 1$ result $>150$ IU/L were identified. Patient results were screened for malignancy; bone pathology; known AMA status; and acute resolving elevated ALP. Unexplained raised ALP were labelled 'possible missed PBC'. Separately antimitochondrial antibody positive (AMA +ve) patients were identified. Patients with a known PBC diagnosis were excluded. Those with a raised ALP trend, +ve AMA and no diagnosis were labelled 'missed PBC'.

Results 2814 patients had raised ALP on $>3$ occasions. 1551 of these (55.2\%) had an AMA result (76 +ve). $1263(44.8 \%)$ had no previous AMA result; of these, 437 (34.6\%) had cancer, $76(6.0 \%)$ underlying bone pathology and $181(14.3 \%)$ had an acute abnormality which resolved. 575 (20.4\% of the total) had no cause identified. In a sample of patients with a 